\title{
Atenção ambulatorial de média complexidade em saúde e reabilitaçáo de pessoas com deficiência física no âmbito da Terapia Ocupacional: reflexóes a partir da prática ${ }^{1}$
}

\author{
Rosé Colom Toldráa, Ana Cristina Fagundes Souto ${ }^{b}$ \\ aProfessora Doutora do Curso de Terapia Ocupacional, Departamento de Fisioterapia, Fonoaudiologia e Terapia \\ Ocupacional, Faculdade de Medicina da Universidade de São Paulo - FMUSP, São Paulo, SP, Brasil \\ ${ }^{\text {b}}$ Terapeuta ocupacional do Curso de Graduação em Terapia Ocupacional, \\ Faculdade de Medicina da Universidade de São Paulo - FMUSP, São Paulo, SP, Brasil
}

\begin{abstract}
Resumo: O presente artigo visa a contribuir para a compreensão da atenção ambulatorial de média complexidade às pessoas com deficiência física e incapacidades que dela decorrem, por meio da prática desenvolvida pela Terapia Ocupacional com essa população no Centro de Docência e Pesquisa do Departamento de Fisioterapia, Fonoaudiologia e Terapia Ocupacional da Universidade de São Paulo, no período de março de 2009 a dezembro de 2010, com periodicidade semanal. Pela escassez de estudos sobre a atenção de média complexidade, foram utilizados os documentos oficiais referentes às políticas públicas da área como referência para a reflexão da prática. Para a análise da experiência foi realizado um estudo qualitativo, do tipo exploratório, a partir de estudo retrospectivo documental dos registros de prontuário de 22 usuários acompanhados. Propõe a utilização de abordagens individuais, grupais e corporais como meio da reapropriação do corpo e do lugar de sujeito produtor da própria saúde. Para a maioria das pessoas, a experiência representou uma possibilidade de acolher e contemplar, mesmo que parcialmente, as necessidades de saúde e reabilitação. A ênfase na estratégia grupal, aliada à corporal, possibilitou fortalecer a capacidade de o usuário cuidar de si, valorizar e integrar o aprendizado adquirido por meio das intervenções com estratégias pessoais adotadas nas atividades do cotidiano.
\end{abstract}

Palavras-chave: Reabilitação, Pessoas com Deficiência Física, Atenção Secundária à Saúde, Grupo, Corpo, Terapia Ocupacional.

\section{Ambulatory care of medium complexity in the health and rehabilitation of persons with physical disabilities within occupational therapy: reflections on practice}

\begin{abstract}
This article aims to contribute to the understanding of medium-complexity ambulatory care delivered to persons with physical impairments and disabilities resulting from these occurrences, through practice developed by the Occupational Therapy with this population at the Center for Teaching and Research of the Department of Physical, Speech and Occupational Therapy at the University of São Paulo, from March 2009 to December 2010, on a weekly basis. Due to the lack of studies on medium complexity health care, official documents of Public Health Policy were used as a guide for reflection on this practice. The research was carried out using a qualitative exploratory approach developed from the documentary study of the records of twenty-two users. It proposes the use of individual, group and body approaches as a means of body reappropriation and capability
\end{abstract}

Autor para correspondência: Rose Colom Toldrá, Faculdade de Medicina da Universidade de São Paulo, Rua Cipotânea, 51, Cidade Universitária, Butantã, CEP 05360-000, São Paulo, SP, Brasil, e-mail: rosetoldra@usp.br

Recebido em 25/11/2011; Revisão em 18/9/2012; Aceito em 15/12/2012. 
development to produce one's own health. For most people, the experience was a chance to receive, albeit partially, their health and rehabilitation needs. The emphasis on the group approach associated with the body approach contributed to improve the user's ability to self-care, and the integration of the knowledge acquired as a result of interventions with strategies adopted in personal daily life activities.

Keywords: Rehabilitation, Disabled Persons, Secondary Health Care, Group, Body, Occupational Therapy.

\section{Introdução}

O presente estudo visa a contribuir para a compreensão das açôes de média complexidade em saúde, realizadas no âmbito da atenção ambulatorial em Terapia Ocupacional junto às pessoas com deficiência física atendidas em projeto vinculado às atividades de pesquisa, ensino e extensão à comunidade, desenvolvida com a participação de estudantes do Curso de Terapia Ocupacional da Universidade de São Paulo.

Para apoiar as reflexôes serão utilizadas referências relativas à Atenção de Média Complexidade, às abordagens grupais e corporais envolvendo usuários e cuidadores/familiares, sob o foco do atendimento terapêutico ocupacional ambulatorial e o contato com a rede de serviços.

Dentre as poucas referências de Atenção de Média Complexidade destacam-se alguns documentos norteadores. O Programa de Atenção à Pessoa Portadora de Deficiência no Sistema Único de Saúde (BRASIL, 1995) apresenta algumas diretrizes para organização e planejamento de serviços e caracteriza o nível 2 de atençáo como tendo o objetivo de

[...] fornecer tratamento em reabilitação, para os casos referendados, mediante recurso de profissional especializado, atuando com critério e base epidemiológica e utilizando tecnologia apropriada para o desenvolvimento de açóes (BRASIL, 1995, p. 29).

Assinala como uma das funçôes na atenção em reabilitação

[...] motivar e orientar a participação dos deficientes e suas famílias, assim como da comunidade, na solução dos seus próprios problemas (BRASIL, 1995, p. 29).

O documento de orientação aos gestores estaduais do Sistema Único de Saúde sobre Assistência de Média e Alta Complexidade relaciona as açóes com os profissionais que compóem esse nível de atenção e refere que a média complexidade ambulatorial é composta
[...] por ações e serviços que visam atender aos principais problemas e agravos de saúde da população, cuja complexidade da assistência na prática clínica demande a disponibilidade de profissionais especializados e a utilizaçáo de recursos tecnológicos, para o apoio diagnóstico e tratamento (BRASIL, 2007a, p. 17).

Dentre os procedimentos que compóem a média complexidade, de acordo com o Sistema de Informaçôes Ambulatoriais (SIA), destacam-se os procedimentos especializados realizados por profissionais médicos, outros profissionais de nível superior e nível médio; terapias especializadas e realização de próteses e órteses (BRASIL, 2007a), assim incluindo a Terapia Ocupacional como uma das categorias profissionais que desenvolvem açóes nesse nível de atenção.

O Ministério da Saúde aponta que o papel da assistência de média complexidade envolve promoção da saúde, prevenção das doenças ou danos, tratamento, reabilitação bem como manutenção e suporte individual e familiar visando o autocuidado. Entretanto, a dificuldade reside em estabelecer parâmetros que possam amparar açóes nesse nível de atenção, pois a visão tem sido tradicionalmente fragmentada e os procedimentos selecionados por exclusão daqueles que não correspondem à Atenção Primária em Saúde, seja pelos custos, seja pela densidade tecnológica envolvida (BRASIL, 2007a).

Além dos aspectos acima referidos, segundo a Política Nacional de Saúde da Pessoa Portadora de Deficiência (BRASIL, 2008a), outro problema a ser considerado é a visão limitada dos serviços sobre como e em que poderiam contribuir para a independência e qualidade de vida das pessoas com deficiência.

A experiência desenvolvida no Programa de Saúde da Família/Qualis Fundação Zerbini (ROCHA, 2006) mostrou a necessidade de integrar os diferentes níveis de atenção, com ênfase na complementaridade das açôes de prevenção e das de reabilitação, realizadas de forma concomitante e em diferentes espaços: no ambulatório, nas unidades de saúde da família, 
nos domicílios e em espaços comunitários, visando atender às demandas de atenção em saúde.

Para melhor compreender a atenção de média complexidade e sua interface com a reabilitaçáo de pessoas com deficiência, cabe resgatar a sua diferenciação dos pressupostos tradicionais do modelo de reabilitação, centrado na dimensão biológica, na doença e na disfunção. Nas últimas décadas, essa abordagem vem sendo reconhecida como insuficiente, na medida em que desconsidera a realidade econômica, política e social vivida por essas pessoas (OLIVER et al., 1999; ROCHA, 1991; SOARES, 1991; TOLDRÁ, 1996; TOLDRÁ; DE SÁ, 2005), já que a atenção demanda uma visão mais abrangente dos diferentes aspectos que interferem no cotidiano e que afetam a qualidade de vida dessa população.

Outra diferenciação, no que concerne ao modelo tradicional, é referida por Jongbloed e Crichton (1990) pela crítica feita à assistência clínica-individual, que não considera os aspectos sociais e políticos que envolvem o contexto. Esses autores propóem a mudança de uma concepção individualista da deficiência para um modelo que considere o meio social, reforçando o impacto positivo dele na qualidade de vida dessa população.

Os questionamentos dos atores envolvidos na atenção às pessoas com deficiência quanto à efetividade das açôes, dada a heterogeneidade de situaçôes sociais, econômicas e culturais, e as demandas do próprio movimento de pessoas com deficiência levaram a uma mudança de paradigma do entendimento da questão, cujo marco de implantação é a Classificação Internacional de Funcionalidade e Incapacidade em Saúde - CIF (ORGANIZAÇÃO..., 2003).

Os conceitos empregados pela CIF vêm auxiliar na compreensão da inter-relação existente entre as condiçóes de saúde e os fatores contextuais, considerando a funcionalidade e a incapacidade do indivíduo como um processo interativo e evolutivo entre a pessoa e o ambiente físico e atitudinal em que ela se insere. Assim, de acordo com a classificação, os fatores do contexto socioambiental podem ser considerados como facilitadores ou como barreiras para a realizaçáo das atividades e participação social. A nova abordagem proposta vem ao encontro de um novo entendimento das práticas relacionadas com a reabilitação e a inclusão social dessas pessoas (BRASIL, 2007b).

Os limites apontados pela política, no contexto atual de mudança de paradigma do modelo médico para um modelo psicossocial, requerem uma compreensão mais abrangente das necessidades dos indivíduos a partir das relaçóes que estabelecem com o contexto de vida. Nesse panorama, as açóes de reabilitação em grupo ganham um papel privilegiado, o que valoriza a reflexão sobre sua utilização no processo de acompanhamento pela Terapia Ocupacional a partir da experiência desenvolvida.

Assim, considera-se que o atendimento em grupo não deve ser visto como perda de qualidade, massificação da atenção ou economia, mas sim como um instrumento eficaz para abordar as vivências intra e interpessoais que afetam de alguma forma a autonomia e a inclusão social das pessoas com deficiência (TOLDRÁ; PÉREZ; MATTA, 2000).

A utilização de grupos é compreendida por diferentes autores como uma ferramenta importante empregada no âmbito da assistência à saúde (BALLARIN, 2007; LERVOLINO; PELICIONI, 2001; MALTA; MERHY, 2010; TOLDRÁ; PÉREZ; MATTA, 2000) por promover e estimular a independência, autonomia, minimizar os efeitos do processo de adoecimento-incapacidade e favorecer a participação social.

Na promoção da saúde, ressaltam-se a construção permanente de grupos com o objetivo de potencializar as capacidades dos sujeitos, as mudanças de comportamentos e as atitudes voltadas para a autonomia e a capacidade de enfrentamento diante de adversidades (SANTOS et al., 2006).

Aliado à abordagem grupal proposta destaca-se o uso de técnicas corporais para atender às demandas de saúde e reabilitação.

As práticas corporais são apontadas pela Política Nacional de Promoção da Saúde (BRASIL, 2010) como importante estratégia de promoçáo de qualidade de vida e prevençáo de adoecimentos a ser implementada no atendimento público e territorializado às comunidades, incluindo as pessoas com deficiência que nelas vivem. Essas práticas corporais, enquanto expressóes individuais e coletivas do movimento corporal, envolvem caminhadas, prescrição de exercícios, práticas lúdicas, esportivas e de lazer (BRASIL, 2010).

Dessa forma, a utilização de instrumentos como os grupos e as práticas corporais, conjugados sob o foco da cotidianeidade que compóe o repertório de intervenção do terapeuta ocupacional, qualifica a atuação profissional junto a essa população, favorecendo uma abordagem ampla e multidimensional que envolve o contexto de vida e as mudanças no cotidiano decorrentes da aquisição de uma deficiência física, aspectos esses que serão foco da análise a seguir. 


\section{Material e métodos}

Trata-se de estudo qualitativo do tipo exploratório (GIL, 2006), dado que a atenção de média complexidade é, ainda, pouco estudada e as publicaçóes sobre o tema são escassas. Portanto, busca-se com este contribuir para a compreensão da assistência desenvolvida junto às pessoas com deficiência física nesse nível de atenção.

Utilizou-se estudo documental efetivado por meio da leitura e análise dos documentos norteadores do SUS e dos registros de atendimento existentes nos prontuários.

A análise documental dos registros de prontuários ocorreu a partir de um estudo retrospectivo dos usuários com deficiência física atendidos no Centro de Docência e Pesquisa do Departamento de Fisioterapia, Fonoaudiologia e Terapia Ocupacional da Universidade de São Paulo entre março de 2009 e dezembro de 2010. Para a realizaçáo do estudo foram incluídos todos os usuários, no total de 22 homens e mulheres entre 25 e 65 anos, com diferentes tipos de deficiência física e/ou alteraçôes cognitivas adquiridas (hemiplegia, hemiparesia, paraparesia e afasia). Foram realizados atendimentos individuais e grupais, com periodicidade semanal e duração de 30 minutos e 1 hora, respectivamente. Além dos usuários do serviço, os familiares/cuidadores foram atendidos semanalmente, em grupo, em sessões de 1 hora.

$\mathrm{O}$ acesso aos prontuários permitiu identificar as demandas dos usuários, o uso da rede de serviços do SUS e refletir acerca das intervençóes realizadas, assim como identificar alguns resultados e limites dessas intervenções.

Desse modo buscou-se por meio de estudo documental "[...] considerar o processo e o contexto social em que realizou-se a construção do registro." (MAY, 2004, p. 228) para, assim, extrair da melhor forma os dados existentes, nos quais se manifestam a realidade social e as versóes dos eventos, sejam relativos aos cuidados com a saúde ou à realização das atividades envolvendo usuários e familiares.

\section{Resultados e discussão}

A proposta desenvolvida foi marcada pela heterogeneidade dos participantes em relação ao diagnóstico, idade, tempo de convivência com essa condiçấo de saúde, problemáticas cotidianas, estrutura e dinâmica familiar e teve como tema agregador entre os participantes as atividades do cotidiano, sejam as desejadas, realizadas ou abandonadas em decorrência da instalação de uma deficiência física e das incapacidades relacionadas ao ambiente físico e relacional.

Mesmo em relação à deficiência física, embora todos apresentassem comprometimentos adquiridos de origem neurológica em decorrência de diferentes diagnósticos, como acidente vascular cerebral, lesão medular e esclerose múltipla, ela se manifestava de formas variadas, considerando o nível das estruturas e funçôes corporais afetadas.

Todos esses fatores apontavam para uma combinação de necessidades individuais - como limitaçôes físicas, cuidado corporal, insuficiência de informaçôes, orientações às famílias/cuidadores - e necessidades coletivas - como falta de transporte adaptado e de participação em atividades de convivência social, pouco acesso aos serviços básicos de saúde e social -, assim como necessidades subjetivas, que demandavam atenção.

Os 22 usuários que participaram da proposta eram em sua maioria provenientes da região do Butantá ou funcionários da USP, com renda obtida por meio de benefícios sociais ou aposentadoria ou, ainda, dependentes de outros familiares. Apresentavam dificuldades de acesso aos serviços de saúde e reabilitação da regiáo tendo em vista a carência de atendimento no âmbito da reabilitação na zona oeste da cidade de Sáo Paulo.

Quanto à faixa etária, observa-se variação de 25 a 65 anos, com significativo número de adultos jovens $(25-45=41 \%)$, ou seja, em fase produtiva, apontando o impacto da aquisição da deficiência na vida profissional e independência econômica da pessoa e família.

O baixo nível de escolaridade (45,4\% com ensino fundamental completo ou incompleto) sugere condiçôes de maior vulnerabilidade no que tange ao acesso à saúde, pois

[...] os efeitos do nível de instrução se manifestam das mais diferentes formas: na percepção dos problemas de saúde; na capacidade de entendimento das informaçôes sobre saúde; na adoção de estilos de vida saudáveis; no consumo e utilização dos serviços de saúde; e na adesão aos procedimentos terapêuticos (BRASIL, 2008b, p. 46).

O grande número de pessoas solteiras, separadas ou viúvas $(63,6 \%)$ pode resultar em reduçáo da rede de suporte cotidiana quando na presença de maior grau de dependência, sendo o "morar só", no caso de pessoas com sequelas de acidente vascular cerebral, um dos fatores associados ao mau prognóstico funcional (GRESHAM et al., 1995 apud WOODSON, 2005). 
As intervençóes terapêuticas foram pautadas na perspectiva da Política de Humanização do SUS (BRASIL, 2004), para a qual humanizar é

[...] ofertar atendimento de qualidade articulando os avanços tecnológicos com acolhimento, com melhoria dos ambientes de cuidado e das condiçóes de trabalho dos profissionais (BRASIL, 2004, p. 6).

Em consonância com essa política centrou-se a atenção nas vivências e potencialidades da pessoa e não na relação queixa-conduta, na medida em que essa dirige o foco para a doença e não estabelece o vínculo fundamental que permite a responsabilidade sanitária, que constitui o ato de saúde (MALTA; MERHY, 2010).

Para o delineamento das propostas tomou-se como parâmetro a singularidade do sujeito e a busca de sua autonomia (MALTA; MERHY, 2010), no sentido da identificação de suas necessidades e do desenvolvimento de propostas que favorecessem suas potencialidades. Por isso, as intervençóes envolveram tanto os usuários como seus familiares e cuidadores, bem como o contato com a rede de serviços, a fim de proporcionar uma atenção voltada às reais necessidades das pessoas, conforme preconizado pelas políticas do setor.

As estratégias adotadas variaram durante o processo de atendimento em Terapia Ocupacional. As intervençôes individuais ocorreram com todos, no ingresso ao programa, visando a identificação das demandas de saúde e reabilitação. Essas intervenções se constituíram como modalidade de acompanhamento por um período intermediário, até ingresso no grupo, ou ainda como acompanhamento constante, considerando as especificidades da demanda que exigia uma atenção mais personalizada. Outro fator que contribuiu para a adoção dessa modalidade foi a impossibilidade de deslocamento para o local de atendimento, pelas restriçóes do serviço de transporte adaptado, bem como falta de disponibilidade de acompanhante e/ou de transporte particular, inviabilizando, em alguns casos, a participação no horário do grupo. A combinação de atendimentos individuais concomitantemente aos grupais foi a estratégia mais amplamente adotada, tendo em vista a complementaridade das abordagens.

A intervenção grupal aconteceu na perspectiva da adesão em saúde, no sentido de um processo educativo que possibilitasse ao indivíduo uma maior compreensão dos processos produtores de incapacidade e maior pró-atividade e envolvimento do sujeito na condução do seu processo de reabilitação, assumindo o "[...] sujeito enquanto sujeito do processo e náo como cumpridor de projetos terapêuticos que não correspondem às suas necessidades de vida." (BERTOLOZZI et al., 2009, p. 1328).

Essa abordagem configurou-se como um instrumento terapêutico que permitiu criar um ambiente de compartilhamento, de trocas de saberes e atos cuidadores (MALTA; MERHY, 2010) entre todos os participantes (usuários, familiares/ cuidadores e profissionais). Esse ambiente favoreceu o aprendizado, contribuindo para o ganho de independência e autonomia, a criação de novos sentidos para a vida, o resgate de atividades de convivência social, o aprendizado e o enfrentamento de situações de discriminação, o estabelecimento de vínculo social e afetivo, a assunção de atividades do cotidiano de acordo com as condiçóes de saúde e uma maior participação social.

Nas dinâmicas grupais e individuais foram adotadas vivências e técnicas corporais de forma $a$ atender às necessidades de reabilitaçáo e ao estabelecimento de uma relação mais saudável com o corpo, conforme trata a Política Nacional de Promoção da Saúde (BRASIL, 2010).

Para o desenvolvimento dessa intervenção partiu-se do pressuposto de que os usuários não são uma "caixa vazia”, adotando-se a acepçáo de Helman (HELMAN, 2003 apud MALTA; MERHY, 2010, p. 600).

Para tanto, valorizaram-se desde o início do atendimento as experiências de cuidados pessoais já adotadas, como prática de caminhada, realização de exercícios, utilização de aprendizados de cuidado, estratégias corporais e manobras aprendidas nos atendimentos com outras áreas profissionais. No processo exploraram-se atividades rotineiras que exigiam uso das capacidades funcionais, como subir escadas, realização de atividades domésticas e de vida diária aliadas à abordagem com o corpo por meio da experimentação de técnicas de consciência corporal e de movimentos baseados prioritariamente no método Self-healing (SCHNEIDER; LARKIN; SCHNEIDER, 1998; SCHNEIDER, 2005) visando o (re)conhecimento do corpo atual e de sua funcionalidade. Essa abordagem integradora difere das aprendizagens adquiridas a partir de uma percepçáo fragmentada do corpo e reforçada pelas açóes que valorizam a lesão cerebral, o lado afetado, a fala comprometida, o sintoma e a disfunção. $\mathrm{Ou}$ seja, tira o foco da atenção na queixa, na doença, como referem Malta e Merhy (2010), identificadas nas experiências prévias de atenção em saúde dos usuários. 
O resgate da aprendizagem adquirida previamente e considerada significativa pelos usuários, integrada às práticas corporais, visava criar condiçôes que facilitassem a eleição de técnicas para o alívio de tensão e dor muscular, prevenção de futuras incapacidades e aumento das capacidades funcionais, possibilitando assim maior adesão a um projeto centrado na melhoria da própria condição de saúde. Como resultado dessa abordagem podem-se destacar maior reconhecimento das próprias capacidades, uso mais equilibrado das funçôes corporais, maior percepção dos limites e menos exposição aos riscos de quedas e de outras condiçóes que pudessem trazer limitações futuras para a realização de atividades e participação social.

Assim, as estratégias desenvolvidas com os usuários estimularam a criação de formas de viver mais ativas e implicadas com a manutenção de melhores condições de saúde, atendendo as políticas do setor (BRASIL, 1995, 2008a, 2010).

Tendo em vista que as repercussóes da deficiência física nas condiçôes de vida não afetam exclusivamente a pessoa com deficiência, mas na maioria das vezes "[...] a família e a sociedade, pois [ela] dependerá de algum cuidador para realizar parcial ou totalmente as atividades de vida diária [...] as funções em casa e no trabalho [...]" (COSTA, 2008 apud DELBONI; MALENGO; SCHMIDT, 2010, p. 167) foi realizado, paralelamente, um acompanhamento com os familiares e cuidadores.

Para eles foram adotadas estratégias que envolveram desde encontros periódicos (semanais ou quinzenais), nos quais se privilegiou o espaço grupal como espaço de troca e aprendizado, até conversas telefônicas e visitas domiciliares. Essas estratégias visaram a conhecer os costumes, a realidade sociofamiliar, o cotidiano da pessoa atendida sob a perspectiva dos principais parceiros (familiares e cuidadores) no que se refere à realização das atividades, dificuldades e alternativas identificadas pelo cuidador, detalhes sobre o quadro clínico e, ainda, oferecer informaçôes do tratamento propriamente dito para, posteriormente, desenvolver orientaçóes adequadas à realidade de cada pessoa.

As orientaçôes foram desenvolvidas de forma verbal, de maneira demonstrativa no caso de dispositivos específicos e técnicas de manuseio, e/ou por meio da utilizaçáo de roteiros escritos e desenhos que explicavam alguns procedimentos. Esses recursos visavam facilitar a comunicação, garantir a utilização das orientaçôes e a continuidade das práticas e aprendizados no contexto de vida, implicando na corresponsabilizaçáo do sujeito e da família/cuidador pelo processo.
As intervençôes com familiares e cuidadores visaram refletir sobre o cuidado cotidiano oferecido, as dificuldades e potencialidades observadas, como forma de complexificação do ato cuidador. A partir disso explorou-se o potencial de convivência na família e o caráter facilitador na utilização de estratégias, para estimular a melhora das condiçốes de realização de atividades e de participação no contexto da família e social. Ou seja, buscou-se motivar e orientar os usuários e familiares para a solução de seus próprios problemas de forma mais autônoma (BRASIL, 1995).

Outro aspecto valorizado na assistência a essa população foi o conhecimento de como os usuários acessavam os serviços do SUS. Procurou-se identificar a trajetória deles pelo sistema de saúde e verificou-se a utilização esporádica ou inexistente da Unidade Básica de Saúde (UBS), havendo tendência à continuidade da assistência no hospital universitário de média complexidade localizado na região oeste ou no hospital de alta complexidade, onde foram atendidos na fase aguda e realizada a posterior continuidade do acompanhamento médico.

Essa realidade mostrou a necessidade de esclarecer sobre o papel da UBS e estimular o usuário no estabelecimento ou fortalecimento de vínculo com a rede de saúde para a manutençáo da assistência, orientando-o quanto ao papel da UBS como porta de entrada preferencial e responsável pelo primeiro nível de atenção à saúde no SUS e assistência, pois o essa acompanhará durante todo o seu ciclo de vida, em todos os processos e nas diferentes fases da vida (BRASIL, 2007a). Essa ação de reabilitação foi considerada relevante para o estabelecimento ou fortalecimento de um sistema de referência e contrarreferência, principalmente no sistema de saúde da regiāo oeste da cidade de Sáo Paulo, onde reside a maioria das pessoas atendidas, de forma a estimular a territorialização.

No contato com a rede pública de serviços de saúde foram realizados encaminhamentos de acordo com as demandas apresentadas pelos usuários, tais como: Ambulatório de Especialidades referência na regiáo e responsável pelo provimento de recursos de tecnologia assistiva e serviços de alta complexidade; a UBS e especialidades profissionais tais como: Fisioterapia, Fonoaudiologia e Psicologia. Destaca-se o encaminhamento realizado para um dos usuários à UBS para a continuidade do cuidado na rede básica, dada a necessidade de materiais de higiene pessoal (sondas, coletores de urina etc.) e de treinamento desse usuário, pela enfermagem, para o seu uso em domicílio, identificando-se nesse caso a falta de suporte para a realização das açôes 
necessárias demandadas pelo usuário. Ainda, no contato com a rede de serviços foram solicitadas informações aos profissionais sobre as intervenções e atendimentos realizados, visando conhecer de forma mais abrangente as diferentes necessidades do acompanhamento terapêutico e de maior grau de integralidade do cuidado, apesar de na maioria das vezes essas solicitaçóes não terem sido respondidas.

A experiência grupal fez emergirem demandas decorrentes do espaço social mais amplo, da possibilidade de transitar e ocupar diferentes papéis, reafirmando a importância da intersetorialidade por meio de encaminhamento aos equipamentos de outros setores, como os Centros de Convivência e os equipamentos de Cultura.

Todas as açôes acima desenvolvidas no âmbito da Atenção de Média Complexidade lançaram mão das tecnologias de trabalho em saúde denominadas por Merhy (2002) de tecnologias leves e leve-duras. As leves referem-se às "tecnologias das relaçôes", ou seja, que envolvem o "espaço intersubjetivo" do profissional de saúde e do usuário apoiadas pelo acolhimento e humanização da atenção, incluindo usuários, familiares e cuidadores. São reconhecidas pelos usuários como espaço de autodescoberta, elaboração de conflitos, melhora da autoestima, onde ocorre a construção das açôes terapêuticas pautadas na elaboração mútua e coletiva. As tecnologias levesduras podem ser identificadas por meio da aplicação de técnicas de avaliação do impacto da deficiência no cotidiano de vida das pessoas, orientaçóes e confecçáo de recursos tecnológicos e uso de técnicas terapêuticas corporais visando à reabilitação e melhoria de qualidade de vida, empregadas para dar sentido e resposta à necessidade dos usuários e para favorecer o resgate do sujeito como corresponsável pela produçáo de sua condiçấo de vida.

\section{Concluindo}

$\mathrm{O}$ atendimento de Terapia Ocupacional representou para a maioria das pessoas uma possibilidade de acolher e contemplar, mesmo que parcialmente, as necessidades de saúde e reabilitação decorrentes da deficiência física adquirida.

As estratégias grupais e corporais adotadas possibilitaram fortalecer a capacidade de o usuário cuidar de si, desenvolver novas estratégias, valorizar as iniciativas dirigidas para a melhoria de qualidade de vida, ressaltando a dimensão cuidadora no âmbito da saúde e reabilitação, centradas nas tecnologias leves e leves-duras, no contexto da atenção ambulatorial de média complexidade.
O processo desencadeado visou a ressignificação do histórico pessoal dos sujeitos alvo de reabilitaçáo a sujeitos produtores de saúde, identificando, refletindo e integrando o aprendizado decorrente das intervenções e das estratégias pessoais desenvolvidas.

\section{Referências}

BALLARIN, M. L. G. S. Abordagens Grupais. In: CAVALCANTI, A.; GALVÃO, C. Terapia Ocupacional: Fundamentação \& Prática. São Paulo: Guanabara Koogan, 2007. p. 38-43.

BERTOLOZZI, M. R. et al. Os conceitos de vulnerabilidade e adesão na Saúde Coletiva. Revista da Escola de Enfermagem da USP, São Paulo, v. 43, p. 1326-30, 2009. Número especial 2. Disponível em: <http://www.scielo.br/pdf/reeusp/v43nspe2/a31v43s2. pdf>. Acesso em: 03 set. 2012. http://dx.doi.org/10.1590/ S0080-62342009000600031

BRASIL. Ministério da Saúde. Secretaria de Assistência à Saúde. Coordenação de Atenção a Grupos Especiais. Programa de Atenção à Saúde da Pessoa Portadora de Deficiência. Brasília: Ministério da Saúde, 1995.

BRASIL. Ministério da Saúde. Secretaria-Executiva. Núcleo Técnico da Política Nacional de Humanização. Humanizasus: Política Nacional de Humanização: a humanização como eixo norteador das práticas de Atenção e gestão em todas as instâncias do SUS. Brasília: Ministério da Saúde, 2004. Disponível em: <http//www.saúde.gov. br/editora>. Acesso em: 15 fev. 2008.

BRASIL. Conselho Nacional de Secretários de Saúde. Comissão Nacional sobre Determinantes Sociais da Saúde - CNDSS. Assistência de Média e Alta Complexidade no SUS. Brasília: CONASS, 2007a.

BRASIL. Ministério da Saúde. Secretaria de Atenção à Saúde. Departamento de Açóes Programáticas Estratégicas. A Pessoa com deficiência e o Sistema Único de Saúde. Brasília: Ministério da Saúde, 2007b.

BRASIL. Ministério da Saúde. Secretaria de Atenção à Saúde. Politica Nacional de Saúde da Pessoa Portadora de Deficiência. Brasília: Ministério da Saúde, 2008a.

BRASIL. Ministério da Saúde. Comissão Nacional sobre Determinantes Sociais da Saúde - CNDSS. As Causas sociais das iniquidades em saúde no Brasil. Brasília: Ministério da Saúde, 2008b. 216 p. Relatório Final da CNDSS. Disponível em: <http://www.cndss.fiocruz. br/pdf/home/relatorio.pdf>. Acesso em: 03 set. 2012.

BRASIL. Ministério da Saúde. Secretaria de Vigilância em Saúde. Secretaria de Atenção à Saúde. Política Nacional de Promoção da Saúde. 3. ed. Brasília: Ministério da Saúde, 2010. Disponível em: <http://bvsms.saude.gov.br/bvs/ publicacoes/politica_nacional_promocao_saude_3ed. pdf>. Acesso em: 22 nov. 2010.

DELBONI, M. C. C.; MALENGO, P. C. M.; SCHMIDT, E. P. R. Relação entre os aspectos das alteraçóes funcionais e seu impacto na qualidade de vida das pessoas com seqüelas de Acidente Vascular Encefálico (AVE). $O$ Mundo da Saúde, São Paulo, v. 34, n. 2, p. 165-175, 2010. 
GIL, A. C. Métodos e Técnicas de Pesquisa Social. Editora Atlas: São Paulo, 2006.

JONGBLOED, L.; CRICHTON, A. A new definition of disability: implications for rehabilitation practice and social policy. Canadian Journal of Occupational Therapy, Toronto, v. 57, n. 1, p. 32, 1990. http://dx.doi. org/10.1177/000841749005700107

LERVOLINO, S. A.; PELICIONI, M. C. F. A utilização do grupo focal como metodologia qualitativa na promoção da saúde. Revista da Escola de Enfermagem da USP, São Paulo, v. 35, n. 2, p. 115-121, jun. 2001. Disponível em: <http://www.scielo.br/scielo.php?script=sci_ arttext \&pid $=$ S0080 - 62342001000200004\&lng=en $\& \mathrm{nrm}=\mathrm{iso}>$. Acesso em: 19 maio 2010. http://dx.doi. org/10.1590/S0080-62342001000200004

MALTA, D. C.; MERHY, E. E. O percurso da linha do cuidado sob a perspectiva das doenças crônicas não transmissíveis. Interface: Comunicação Saúde e Educação, Botucatu, v. 14, n. 34, p. 593-605, 2010. http://dx.doi. org/10.1590/S1414-32832010005000010

MAY, T. Pesquisa social - questóes métodos e processos. Porto Alegre: Artmed, 2004.

MERHY, E. E. Saúde: a cartografia do trabalho vivo. São Paulo: Hucitec, 2002.

OLIVER, F. S. et al. Reabilitação Baseada na Comunidade - discutindo estratégias de ação no contexto sociocultural. Revista de Terapia Ocupacional da USP, São Paulo, v. 10, n. 1, p. 1-10, 1999.

ORGANIZAÇÃO MUNDIAL DA SAÚDE - OMS. Centro Colaborador da Organização Mundial da Saúde para a Família de Classificaçôes Internacionais. CIF: Classificação Internacional de Funcionalidade, Incapacidade e Saúde. São Paulo: EdUSP, 2003.

ROCHA, E. F. Corpo Deficiente: em busca da reabilitação? Uma reflexão a partir da ótica das pessoas com deficiência física. 1991. 310 f. Dissertação (Mestrado em Psicologia)Instituto de Psicologia, Universidade de São Paulo, São Paulo, 1991.

ROCHA, E. F. Reabilitação de Pessoas com Deficiência: a intervenção em discussão. São Paulo: Roca, 2006.

SANTOS, L. M. et al. Grupos de promoção à saúde no desenvolvimento da autonomia, condiçôes de vida e saúde. Revista de Saúde Pública, São Paulo, v. 40, n. 2, p. 346-352, 2006. http://dx.doi.org/10.1590/ S0034-89102006000200024

SCHNEIDER, M.; LARKIN, M.; SCHNEIDER, D. Manual de Auto-cura: Método Self-Healing 1a Parte. São Paulo: Ed. Triom, 1998.

SCHNEIDER, M. Movimento para a auto-cura. São Paulo: Cultrix, 2005.

SOARES, L. B. T. Terapia Ocupacional: lógica do capital ou do trabalho? São Paulo: Hucitec, 1991.

TOLDRÁ, R. C. Aprender a Vivir: la construcción de la identidad de la persona con discapacidad física. 1996. 393 f. Tese (Doutorado em Sociología)-Universitat de Barcelona, Barcelona, 1996.

TOLDRÁ, R. C.; DE SÁ, M. J. C. N. A visão do usuário de um serviço de terapia ocupacional: em busca da qualidade da assistência. Revista de Terapia Ocupacional da USP, São Paulo, v.16, n.2, p.90-96, maio/ago. 2005.

TOLDRÁ, R. C.; PÉREZ, M. A. G.; MATTA, M. A. P. Acesso e qualidade da atenção à pessoa portadora de deficiência física em serviços públicos de saúde do município de Campinas. Revista de Terapia Ocupacional da USP, São Paulo, v. 11, n. 2-3, p. 72-8, maio/dez. 2000.

WOODSON, A. M. Acidente Vascular Cerebral. In: TROMBLY, C. A.; RADOMSKI, M. V. Terapia Ocupacional para disfunções físicas. 5. ed. São Paulo: Livraria Editora Santos, 2005. cap. 38, p. 817-854.

\section{Contribuição dos Autores}

A concepção da pesquisa e do texto manuscrito é de Rosé Colom Toldrá. A redação do manuscrito, análises e organização das fontes foi feita conjuntamente pelas duas autoras: Rosé Colom Toldrá e Ana Cristina Fagundes Souto.

\section{Notas}

${ }^{1}$ Parte da pesquisa Atenção à Saúde e reabilitação no contexto da Assistência de Média Complexidade no âmbito da Terapia Ocupacional: perfil, demandas e açóes, aprovada pelo Comitê de Ética em Pesquisa em Seres Humanos da Faculdade de Medicina da USP sob protocolo número 243/11. 